\title{
Magnetic control of the valley degree of freedom of massive Dirac fermions with application to transition metal dichalcogenides
}

\author{
Tianyi Cai, ${ }^{1}$ Shengyuan A. Yang,,${ }^{2, *}$ Xiao Li, ${ }^{3}$ Fan Zhang, ${ }^{4}$ Junren Shi, ${ }^{5}$ Wang Yao, ${ }^{6}$ and Qian Niu ${ }^{3,5}$ \\ ${ }^{1}$ School of Physical Science and Technology, Soochow University, Suzhou 215006, China \\ ${ }^{2}$ Engineering Product Development, Singapore University of Technology and Design, Singapore 138682, Singapore \\ ${ }^{3}$ Department of Physics, The University of Texas at Austin, Austin, Texas 78712, USA \\ ${ }^{4}$ Department of Physics and Astronomy, University of Pennsylvania, Philadelphia, Pennsylvania 19104, USA \\ ${ }^{5}$ International Center for Quantum Materials, Peking University, Beijing 100871, China \\ ${ }^{6}$ Department of Physics and Center for Theoretical and Computational Physics, The University of Hong Kong, Hong Kong, China
}

(Received 29 August 2013; published 25 September 2013)

\begin{abstract}
We study the valley-dependent magnetic and transport properties of massive Dirac fermions in multivalley systems such as transition metal dichalcogenides. The asymmetry of the zeroth Landau level between valleys and the enhanced magnetic susceptibility can be attributed to the different orbital magnetic moment tied with each valley. This allows the valley polarization to be controlled by tuning the external magnetic field and the doping level. As a result of this magnetic-field-induced valley polarization, there exists an extra contribution to the ordinary Hall effect. All these effects can be captured by a low-energy effective theory with a valley-orbit coupling term.
\end{abstract}

DOI: 10.1103/PhysRevB.88.115140

PACS number(s): 71.70.Di, 73.43.-f, 73.50.-h

\section{INTRODUCTION}

Many crystalline materials have multiple energy extremum points near the Fermi level in reciprocal space, which are related to each other through symmetry operations and are referred to as valleys. A well-known example is $\mathrm{Si}$, which has six valleys at its conduction-band edge in the Brillouin zone. Similar to spin, the valley labeling constitutes a discrete degree of freedom. Therefore, it was proposed that the valley degree of freedom could be used for information coding and transmission, giving rise to an active research field called valleytronics. $^{1-8}$

To have a successful valleytronics application, there are at least two basic requirements. The first is the ability to generate and control the valley polarization, and the second is the ability to detect the valley polarization. For conventional semiconductor materials such as $\mathrm{Si}$, it is difficult to distinguish different valleys. The situation changes with the advent of novel two-dimensional (2D) materials which support massive Dirac fermion excitations. Examples include graphene ${ }^{9-11}$ with sublattice symmetry breaking, silicene, transition metal dichalcogenides, ${ }^{12-15}$ etc. In these systems, there are two inequivalent valleys $K$ and $K^{\prime}$ located at the corners of the hexagonal Brillouin zone. The special feature of the massive Dirac fermion-type excitation is that each valley has a definite chirality arising from its strong pseudospin-orbit coupling. ${ }^{2}$ More importantly, the chiralities of the two valleys are opposite to each other, which is imposed by the time-reversal and inversion symmetries. This leads to possible practical ways to differentiate the two valleys and to address them individually. ${ }^{2,4}$

Of the examples mentioned above, 2D transition metal dichalcogenides (TMDs) are especially interesting and have attracted a lot of attention recently. ${ }^{16-31}$ It has been found that when thinned down to a single layer, several members of this class of materials undergo a transition from an indirect band gap to a direct band gap with a gap size of $1-2 \mathrm{eV}$, which is suitable for optical manipulations. ${ }^{16-18}$ It has been successfully demonstrated that the excitonic valley polarization and coherence in 2D TMDs can be generated by pumping with circularly polarized light and linearly polarized light, respectively. ${ }^{19,21-25}$ The optically generated excitonic states could be manipulated electrically, ${ }^{26,27}$ and have a long spin coherence time due to the large valley separation and the large spin splitting in the valence band. ${ }^{19,25}$ The field-effect transistors with a single layer of $\mathrm{MoS}_{2}$ have also been fabricated, and the mobility can be enhanced to $500 \mathrm{~cm}^{2} / \mathrm{Vs},{ }^{29-31}$ with an excellent current on/off ratio.

Motivated by this recent progress, we explore the possibility of controlling the valley degree of freedom in TMDs through magnetic means. We show that the zeroth Landau level anomaly, which was found previously in $\mathrm{MoS}_{2}$ (Ref. 32), and the enhanced magnetic susceptibility can be attributed to the valley-contrasting orbital magnetic moments. With this property, we could generate valley polarization of carriers by using an external magnetic field. Furthermore, this induced valley polarization would in turn produce an extra contribution to the ordinary Hall effect which can be detected experimentally. A simple effective theory is proposed to describe the dynamics of such valley-orbit coupled systems. These findings may open a new route for valleytronics applications.

This paper is organized as follows. In Sec. II we discuss the simplest model of massive Dirac fermions, which serves as the generic building blocks for the more realistic models. In Sec. III we apply the results from Sec. II to study the TMD materials and discuss their Landau level structures and magnetic susceptibility. In Sec. IV we show that the magnetic field can be used to control the valley polarization. In Sec. V we predict that this valley polarization leads to an extra contribution in the charge Hall transport. Some discussions and a summary are presented in Sec. VI.

\section{SIMPLE MODEL OF MASSIVE DIRAC FERMIONS}

In this section, we first present a heuristic discussion of the simplest model for a massive Dirac fermion with valley degrees 
of freedom. In the absence of external fields, the model can be written as

$$
H_{\mathrm{D}}=\hbar v\left(\tau_{z} k_{x} \sigma_{x}+k_{y} \sigma_{y}\right)+\Delta \sigma_{z},
$$

where $v$ is a material-specific Fermi velocity, $k_{x}$ and $k_{y}$ are the two components of the wave vector measured from the Dirac point, $\sigma$ 's are the Pauli matrices typically representing a pseudospin from the sublattice or orbital degrees of freedom, and $\tau_{z}= \pm 1$ is the valley index labeling the two inequivalent valleys. The form of the Hamiltonian is generic for several 2D materials we are interested in, including the TMD materials. These materials usually have a honeycomb lattice structure when viewed from the top. The two valleys occur at $K$ and $K^{\prime}$ points at the corners of the hexagonal Brillouin zone, and are related to each other by the time-reversal and inversion symmetries.

The first term in $H_{0}$ shows a strong pseudospin-orbit coupling and the second term is the mass term. If $\Delta \rightarrow 0$, the particle becomes massless and the Hamiltonian can be used to describe the graphene. For a finite $\Delta$, it opens a gap of $2 \Delta$ in the spectrum.

The two valleys have different chiralities. This can be understood by tracking the pseudospin orientation when an electron moves around a fixed energy contour enclosing a Dirac point. Consider an electron going around the states (counterclockwise) with a fixed energy $\varepsilon>2 \Delta$ and returning to its starting point. Its pseudospin $\sigma$ would rotate by $+2 \pi$ for the $K$ valley $\left(\tau_{z}=+1\right)$, while it would rotate by $-2 \pi$ for the $K^{\prime}$ valley $\left(\tau_{z}=-1\right)$. This difference in the chirality or the winding numbers manifests in many important electronic properties such as the Berry curvature and the orbital magnetic moment. 2,6

\section{A. Asymmetric Landau levels}

The effect of an external magnetic field (oriented in the $z$ direction, i.e., perpendicular to the plane) can be taken into account through the Peierls substitution of $\boldsymbol{k}$ by $\boldsymbol{\pi}=$ $\boldsymbol{k}+\boldsymbol{e} \boldsymbol{A} / \hbar$, where $\boldsymbol{A}$ is the vector potential. Here we neglect the Zeeman energy term, which is at least one order of magnitude smaller than the cyclotron energy. ${ }^{32}$ Hence in the remaining part of this section, we shall neglect the spin degeneracy. Following the standard procedure for the Landau level quantization, we define the operators $\pi_{ \pm}=\pi_{x} \pm i \pi_{y}$ which satisfy the commutation relation $\left[\pi_{-}, \pi_{+}\right]=2 e B / \hbar$, where $B$ is the magnitude of the magnetic field. Hence we could define the bosonic ladder operators $b^{\dagger}$ and $b$ as $b^{\dagger}=$ $\left(l_{B} / \sqrt{2}\right) \pi_{+}, \quad b=\left(l_{B} / \sqrt{2}\right) \pi_{-}$, where $l_{B}=\sqrt{\hbar /(e B)}$ is the magnetic length. These ladder operators satisfy the relations $b|n\rangle=\sqrt{n}|n-1\rangle, b|0\rangle=0$, where $|n\rangle(n=0,1,2, \ldots)$ are the Landau level eigenstates for a conventional $2 \mathrm{D}$ electron gas.

The spectrum can be easily solved on the basis of $|n\rangle$ 's. The resulting Landau levels are

$$
\varepsilon_{n, \pm}=\tau_{z} \Delta \delta_{n, 0} \pm \sqrt{\Delta^{2}+n \hbar^{2} \omega_{c}^{2}}\left(1-\delta_{n, 0}\right),
$$

where $\omega_{c}=\sqrt{2} v / l_{B}, \delta$ is the Kronecker delta function, and $n$ is an integer $\geqslant 0$.

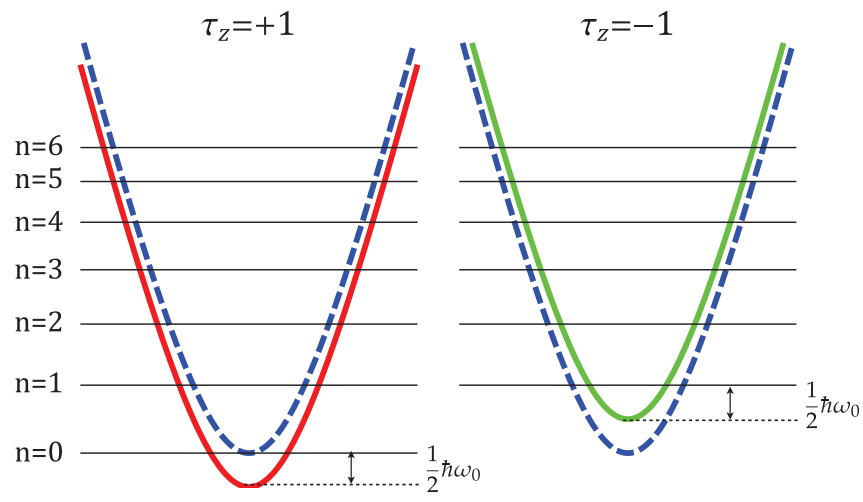

FIG. 1. (Color online) The Landau levels from exact quantum calculations for the conduction band of the two valleys, shown as solid horizontal lines. The original band dispersion in the absence of fields is shown as dashed blue curves. The solid curves show the band dispersion shifted by the Zeeman-like coupling between magnetic moment $\boldsymbol{m}(\boldsymbol{k})$ and the magnetic field. It can be seen that the first Landau level is at $\hbar \omega_{0} / 2$ above the bottom of the shifted band, where $\hbar \omega_{0}$ is the cyclotron energy of the first Landau level.

We observe that the Landau level spacing is not uniform (see Fig. 1). The Landau levels with $n \geqslant 1$ are aligned between the two valleys. However, the zeroth Landau level with $n=0$ is not located at the zero energy, and for different valleys its position shifts in opposite directions. Note that the zeroth Landau level for the $\tau_{z}=+1$ valley is at the same energy of the original conduction-band bottom at zero field, while for the other valley there is no Landau level at this energy. The spacing between the zeroth and the first Landau level in the $\tau_{z}=+1$ valley is

$$
\delta \varepsilon=\sqrt{\Delta^{2}+\hbar^{2} \omega_{c}^{2}}-\Delta \approx \frac{e \hbar v^{2}}{\Delta} B,
$$

where in the second step we assume the gap is much larger than the cyclotron energy.

This peculiar asymmetric behavior can be traced to the chirality difference between the two valleys and can be easily explained in the semiclassical theory of Bloch electrons. If we construct an electron wave packet near the valley center, due to the pseudospin-orbit coupling, it is self-rotating, hence producing an intrinsic orbital magnetic moment. The general expression is given by ${ }^{33-35}$

$$
\boldsymbol{m}(\boldsymbol{k})=-i \frac{e}{2 \hbar}\left\langle\nabla_{\boldsymbol{k}} u|\times[H(\boldsymbol{k})-\varepsilon(\boldsymbol{k})]| \nabla_{\boldsymbol{k}} u\right\rangle,
$$

where $|u\rangle$ is the periodic part of the Bloch eigenstate, $H(\boldsymbol{k})$ is the Bloch Hamiltonian, and $\varepsilon(\boldsymbol{k})$ is the band energy. Generally speaking, the orbital magnetic moment is large for states near gaps caused by (pseudo-)spin-orbit coupling. For our present case, a direct calculation yields

$$
\boldsymbol{m}(\boldsymbol{k})=\tau_{z} \frac{e \hbar v^{2} \Delta}{2\left(\Delta^{2}+\hbar^{2} v^{2} k^{2}\right)} \hat{\boldsymbol{z}} .
$$

This moment is largest at the band edges $\pm \Delta$. For example, at the conduction-band bottom, it is given by

$$
\boldsymbol{m}=\tau_{z} \frac{e \hbar}{2 m_{e}^{*}} \hat{z} \quad \text { with } \quad m_{e}^{*}=\frac{\Delta}{v^{2}} .
$$


Here we express the moment in a form similar to that for the Bohr magneton. The difference is that now the bare electron mass is replaced by an effective mass $m_{e}^{*}$ determined by the band parameters. It is noted that the magnitude of this moment is proportional to $\Delta^{-1}$, i.e., the moment becomes larger when the gap is smaller. The most important feature for our purpose is that it takes different signs for the two valleys. This is a manifestation of the valley-dependent chirality of the two valleys we mentioned before, because chirality can be viewed as representing a sense of rotation of the carrier. It is an important property of the electronic band structure.

In the presence of an external magnetic field, the wavepacket energy would be shifted by $-\boldsymbol{m} \cdot \boldsymbol{B} .{ }^{33}$ We plot the shifted bands in Fig. 1. The bands at the two valleys are shifted in opposite directions. In particular, the band edges are shifted by

$$
\varepsilon_{m}=-\tau_{z} \frac{e \hbar v^{2}}{2 \Delta} B .
$$

Its magnitude is equal to $\delta \varepsilon / 2$, i.e., half the spacing between the zeroth and the first Landau levels. Now we can see that with respect to the shifted bands, the Landau levels at both valleys start at half of the cyclotron energy above the band edges, which is just the familiar result for the 2D free-electron gas. This implies that the behavior of the present system under a magnetic field can be understood using a 2D electron gas with a valley-contrasting intrinsic magnetic moment. This point of view will be further supported in the next subsection when we study the magnetic susceptibility. In fact, the entire Landau level spectrum could be obtained in the semiclassical theory through the semiclassical quantization procedure as outlined in the Appendix.

\section{B. Enhanced magnetic susceptibility}

The magnetic susceptibility captures the collective response of the system to the external magnetic field. It can be calculated from the thermodynamic potential

$$
F=-\frac{1}{\beta} \frac{e B}{h} \operatorname{Tr}\left\{\ln \left[1+e^{\beta(\mu-\hat{H})}\right]\right\},
$$

where $\beta=1 /\left(k_{B} T\right)$ is the inverse temperature and $h$ is the Planck constant. The magnetic susceptibility can be extracted as $\chi=-\left(\partial^{2} F / \partial B^{2}\right)_{\mu, B \rightarrow 0}$. Substituting in the Landau level spectrum, we can expand $F$ as a power series in the field strength $B$ and obtain an analytical expression of susceptibility: ${ }^{36}$

$$
\chi_{0}(\mu ; \Delta)=-\frac{e^{2} v^{2}}{6 \pi} \frac{\sinh (\beta \Delta)}{\Delta[\cosh (\beta \mu)+\cosh (\beta \Delta)]} .
$$

In the limit of zero gap $\Delta \rightarrow 0$, we have

$$
\lim _{\Delta \rightarrow 0} \chi_{0}=-\frac{e^{2} v^{2}}{6 \pi} \frac{\beta}{1+\cosh (\beta \mu)},
$$

which recovers the old result discussed in the context of graphene. ${ }^{37,38}$ We observe that the $\chi_{0}$ in the zero gap case has a large negative peak at $\mu=0$, and it diverges as $T \rightarrow 0$. The divergence is removed when the gap opens up, but the large diamagnetic dip is still visible and it gets broadened in energy as the gap increases (see Fig. 2). It is interesting to note

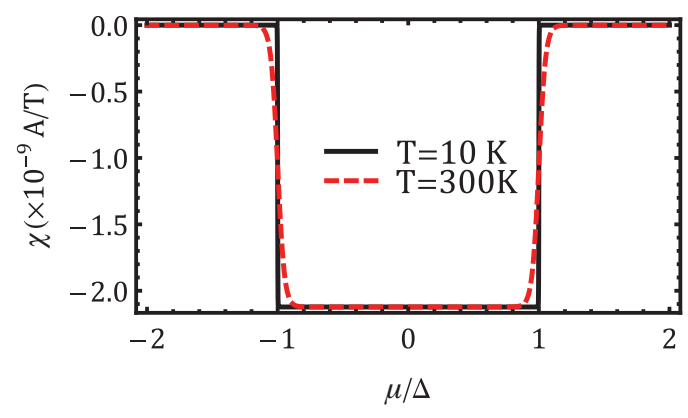

FIG. 2. (Color online) Magnetic susceptibility as a function of the chemical potential $\mu$. Two different temperatures of $T=10$ and $300 \mathrm{~K}$ are taken. Here we take $v=0.5 \times 10^{6} \mathrm{~m} / \mathrm{s}$.

the integral of susceptibility over the chemical potential,

$$
\int_{-\infty}^{+\infty} \chi_{0}(\mu) d \mu=-\frac{e^{2} v^{2}}{3 \pi}
$$

which is independent of both the gap size and the temperature. At the zero-temperature limit, the susceptibility becomes a square well shape, and completely vanishes outside the gap. ${ }^{39}$

The sudden jump of magnetic susceptibility at the band edges in fact signifies a large paramagnetic response from the carriers. Indeed, if we calculate the magnetic susceptibility from the Landau levels above the gap, then the contribution from the conduction-band carriers can be obtained as

$$
\chi_{0}^{c}(\mu)=\frac{e^{2} v^{2}}{6 \pi} \frac{1}{\Delta} \frac{1}{1+e^{-\beta(\mu-\Delta)}},
$$

which shows a large paramagnetic response from the conduction-band electrons.

The large paramagnetic response at the band edge can also be understood in our semiclassical picture mentioned before. At the conduction-band bottom, the system can be viewed as a 2D gas of electron wave packets with magnetic moments $\boldsymbol{m}$. In the presence of an external magnetic field, the resulting magnetic response is well known. It has a net paramagnetic response from the difference between the Pauli paramagnetism and the Landau diamagnetism. ${ }^{40}$ We have checked that the result just recovers Eq. (12) above. Similarly, the large diamagnetic response in the gap can be attributed to the orbital magnetic moments concentrated at the valence-band top. In the zero gap limit, $m_{e}^{*} \rightarrow 0$ and the moment diverges at the band edges, which results in the singular behavior of the magnetic susceptibility. ${ }^{37}$

\section{MAGNETIC RESPONSE OF TRANSITION METAL DICHALCOGENIDES}

In this section, we apply the knowledge obtained from the preceding section to study the magnetic response of a monolayer TMD material. Physically interesting TMD materials have the form of $M X_{2}(M=\mathrm{Mo}, \mathrm{W} ; X=\mathrm{S}, \mathrm{Se}){ }^{20}$ As we mentioned earlier, these materials have direct band gaps at $K$ and $K^{\prime}$ points. They also have a large spin-orbit coupling induced spin splitting in the valence band. The electronic properties near the band edges can be described by the effective Hamiltonian $^{19}$

$$
H=\hbar v\left(\tau_{z} k_{x} \sigma_{x}+k_{y} \sigma_{y}\right)+\Delta \sigma_{z}-\lambda \tau_{z} s_{z} \sigma_{z}+\lambda \tau_{z} s_{z} .
$$


Note that the first two terms are the same as those in our simple massive Dirac model Eq. (1). Now the pseudospin $\sigma$ represents the space of two relevant orbitals $d_{z^{2}}$ and $d_{x^{2}-y^{2}}+i d_{x y}$. The last two terms represent the effect of spin-orbital coupling induced spin splitting. It splits the valence-band top into two spin-polarized bands. $\lambda$ is the coupling strength and $s_{z}= \pm 1$ is for the $z$ component of real spin.

We note that because of the extra spin degrees of freedom, the model in fact consists of two copies of the simple model (1) with different band gaps (masses) depending on the "flavor" index $\tau_{z} s_{z}$. The gap is $2(\Delta-\lambda)$ for $\tau_{z} s_{z}=+1$ and $2(\Delta+\lambda)$ for $\tau_{z} s_{z}=-1$. In this way, the spin and valley are coupled together. The valence-band top in the $K$ valley is polarized with spin-up while in the $K^{\prime}$ valley it is polarized with spin-down. Accordingly, there are two sets of Landau levels which can be written in a compact way as ${ }^{32}$

$$
\varepsilon_{n, \pm}=\lambda \tau_{z} s_{z} \pm \sqrt{\tilde{\Delta}^{2}+n \hbar^{2} \omega_{c}^{2}}
$$

where $\tilde{\Delta}\left(\tau_{z}, s_{z}\right)=\Delta-\lambda \tau_{z} s_{z}$ is the flavor-dependent mass and $n=0,1,2 \ldots$ is a non-negative integer. Again the asymmetric Landau level structure between the two valleys can be observed. It is also interesting to note that the Landau levels near the valence-band top at the $K^{\prime}$ valley are spin-polarized (see Fig. 3).

The magnetic susceptibility can be directly read out by combining the contributions from the two copies of massive Dirac bands:

$$
\chi(\mu)=\chi_{0}(\mu-\lambda ; \Delta-\lambda)+\chi_{0}(\mu+\lambda ; \Delta+\lambda),
$$

where $\chi_{0}(x ; y)$ is defined in Eq. (9). Due to the orbital magnetic moments of carriers at the band edges, again a sudden change of orbital magnetic susceptibility is expected there. This behavior is shown in Fig. 4. And because here we have two copies of massive Dirac bands with different gap sizes, there appears a two-step feature in the susceptibility curve plotted as a function of the chemical potential. The steep change feature is going to be smoothed out as temperature increases.
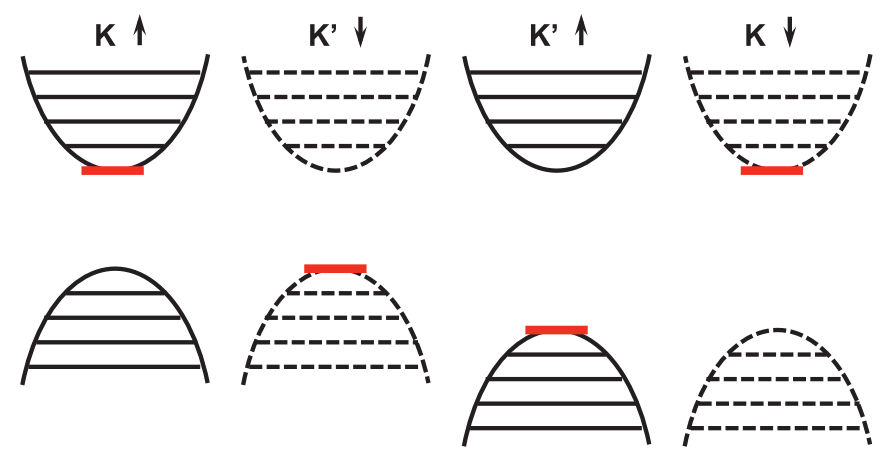

FIG. 3. (Color online) Landau levels for the massive Dirac fermions in transition metal dichalcogenides. Solid (dashed) curves represent spin-up (-down) bands, while the parallel lines represent their Landau levels. The four red lines represent the location of the $n=0$ Landau levels.

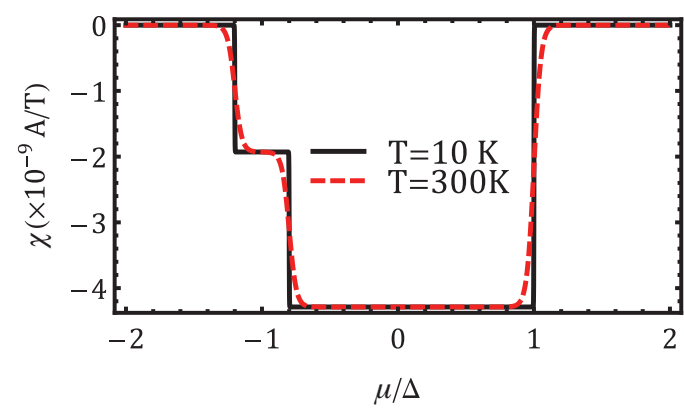

FIG. 4. (Color online) Magnetic susceptibility of typical TMD materials. Two different temperatures of $T=10$ and $300 \mathrm{~K}$ are taken. Here we take the spin-orbit coupling strength to be $\lambda=0.1 \Delta$ and $v=0.5 \times 10^{6} \mathrm{~m} / \mathrm{s}$.

\section{MAGNETIC-FIELD-INDUCED VALLEY POLARIZATION}

A necessary requirement for valleytronics applications is the ability to generate and control the valley polarization. It has been shown that the valley polarization in TMDs can be generated by a circularly polarized light which couples to the orbital magnetic moment. Here we show that due to the valleycontrasting orbital magnetic moment, we can also control the valley polarization in TMDs by an applied magnetic field.

First analogous to the spin polarization, we can define the valley polarization of carriers as

$$
P_{\mathrm{v}}=\frac{n_{+}-n_{-}}{n}
$$

where $n_{ \pm}$is the density of electrons in the valley with index $\tau_{z}= \pm 1$, and $n=n_{+}+n_{-}$is the total carrier density. In an external magnetic field, $n_{ \pm}$can be calculated from the filling of the Landau levels in the two valleys. Now it is easy to see that a finite valley polarization must be present due to the asymmetric Landau level structure of the two valleys.

Considering the $n$-doped case, because the Landau levels (there are two levels from the spin degeneracy) at $+\Delta$ for the $K$ valley have no counterpart at the $K^{\prime}$ valley (and hence for low doping only these two lowest levels are filled), we can achieve $100 \%$ valley polarization. If we keep on increasing the doping, higher Landau levels from both valleys are going to be filled. The valley polarization decreases and approaches zero as $n \rightarrow \infty$. The situation is similar for the $p$-doped case. More interestingly, because of the spin splitting at the valence-band top, the valley polarization is also the spin polarization. Specifically, at a low doping level, only the Landau level at $-\Delta+2 \lambda$ at the $K^{\prime}$ valley is occupied by holes with spin-up. Moreover, for both cases, the valley polarization can be reversed by simply reversing the direction of the magnetic field.

Let us take the simple $T=0$ limit. Then the polarization can be obtained by simply counting the Landau levels. For the $n$-doped case, the variation of $P_{\mathrm{v}}$ as a function of the conduction-band electron density $n_{e}$ is given by (note that there are two spin-degenerate zeroth levels at the $K$ valley)

$$
P_{\mathrm{v}}=\Theta\left(2-v_{e}\right)+\frac{2}{v_{e}} \Theta\left(v_{e}-2\right),
$$




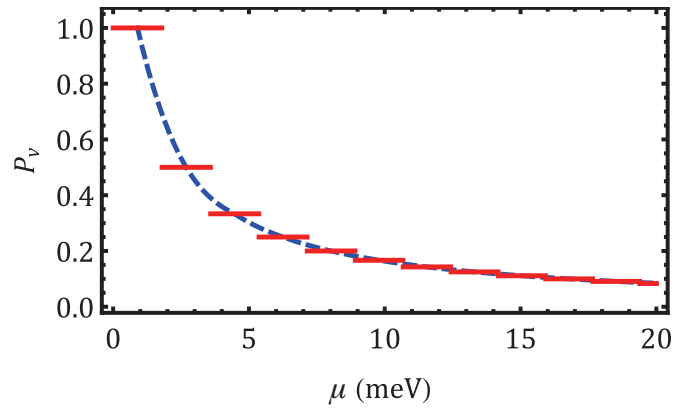

FIG. 5. (Color online) The variation of valley polarization $P_{\mathrm{v}}$ with the chemical potential $\mu$. Here we take $v=0.5 \times 10^{6} \mathrm{~m} / \mathrm{s}, \Delta=0.5$ $\mathrm{eV}$, the spin-orbit coupling strength $\lambda=0.05 \mathrm{eV}$, and the magnetic field $B=5 \mathrm{~T}$. The red steps represent exact quantum calculations, while the blue dashed curve represents the semiclassical results. The chemical potential is measured from the conduction-band $n=0$ Landau level.

where $\Theta$ is the step function, and $v_{e}=h n_{e} /(e B)$ counts the filling of Landau levels. Similarly for the weakly $p$-doped case, i.e., when the Fermi level is above the lower spin-split band,

$$
P_{\mathrm{v}}=-\Theta\left(1-v_{h}\right)-\frac{1}{v_{h}} \Theta\left(v_{h}-1\right) .
$$

Here $v_{h}=h n_{h} /(e B)$ with $n_{h}$ being the hole density.

The valley polarization as a function of the chemical potential would show a series of steps at low temperatures. Again taking the $T=0$ limit, we have for the $n$-doped case

$$
P_{\mathrm{v}}=\frac{1}{1+m}, \quad E_{m} \leqslant \mu<E_{m+1},
$$

where $E_{m}$ is the $m$ th Landau level energy for the conduction band including both flavors $\tau_{z} s_{z}= \pm 1$, and $E_{0}=+\Delta$ is the zeroth Landau level energy at the $K$ valley. The result is plotted in Fig. 5. For the weakly $p$-doped case, the valley polarization is given by

$$
P_{\mathrm{v}}=-\frac{1}{1+2 m}, \quad E_{m+1}<\mu \leqslant E_{m},
$$

where we count the Landau levels from $\mu=0$ with decreasing energy and $E_{0}=-\Delta+\lambda$ is the energy of the zeroth level at the $K^{\prime}$ valley. At finite temperature, the sharp changes of the above functional dependence of $P_{\mathrm{v}}$ are going to be smoothed out, but the main features should be maintained.

The valley polarization can also be calculated using the semiclassical theory, in which the electron density from a given valley is obtained by integrating the Berry curvature modified density of states, ${ }^{41}$

$$
n\left(\mu, \tau_{z}, s_{z}\right)=\int^{\mu} \frac{d^{2} k}{(2 \pi)^{2}}\left(1+\frac{\boldsymbol{B} \cdot \boldsymbol{\Omega}}{\hbar}\right) f\left(k, \tau_{z}, s_{z}\right),
$$

where the Berry curvature $\boldsymbol{\Omega}(\boldsymbol{k})=i\left\langle\nabla_{\boldsymbol{k}} u|\times| \nabla_{\boldsymbol{k}} u\right\rangle$ is also an intrinsic band property like the orbital magnetic moment, and $f$ is the Fermi distribution function. The effect of the external field is in the shift of the bands $f=f[\varepsilon(k)-m(k) B](\varepsilon$ and $m$ also depend on $\tau_{z}$ and $s_{z}$ ). The resulting valley polarization is a smooth curve going through all the steps of the exact quantum result Eq. (19) (see Fig. 5). It is a good approximation in the low-field regime, as it should be from the condition of validity of the semiclassical theory.

\section{HALL TRANSPORT FROM VALLEY POLARIZATION}

It is now clear that even in the absence of the external magnetic field, transverse motion of the carriers could be induced due to the Berry curvature, which acts like an effective magnetic field in reciprocal space. ${ }^{42,43}$ Like the orbital magnetic moment, the Berry curvature is also related to the chirality of the band. For the present model of TMDs, the broken inversion symmetry leads to a nontrivial momentumspace Berry curvature which reads ${ }^{19,32}$

$$
\Omega_{c}\left(k, \tau_{z}, s_{z}\right)=-\tau_{z} \frac{\hbar^{2} v^{2} \tilde{\Delta}}{2\left(\tilde{\Delta}^{2}+\hbar^{2} v^{2} k^{2}\right)^{3 / 2}} .
$$

The appearance of the factor of $\tau_{z}$ indicates it is also a valleycontrasting property. For the valence band, the Berry curvature has the opposite sign, $\Omega_{v}\left(k, \tau_{z}, s_{z}\right)=-\Omega_{c}\left(k, \tau_{z}, s_{z}\right)$.

In the presence of an in-plane electric field, this Berry curvature leads to the transverse motion of the carriers. Its integral over the occupied states gives an intrinsic contribution to the Hall conductivity, ${ }^{42,43}$

$$
\sigma_{\mathrm{H}}^{\mathrm{int}}=\frac{e^{2}}{\hbar} \sum_{\tau_{z}, s_{z}} \int \frac{d^{2} k}{(2 \pi)^{2}} f\left(k, \tau_{z}, s_{z}\right) \Omega\left(k, \tau_{z}, s_{z}\right),
$$

where $f$ is the Fermi-Dirac distribution function. Disorder scattering also contributes to the Hall transport. There is an important side-jump contribution which is proportional to the Berry curvature at the Fermi surface. ${ }^{44}$ In the following, we assume the scattering is of Gaussian white-noise type ${ }^{45}$ and we disregard the intervalley scattering, which requires a large momentum transfer.

We first consider a single copy of a massive Dirac fermion as in Eq. (1). For the $n$-doped case $(\mu>\Delta)$, the Hall conductivity for each valley has been obtained before as ${ }^{2,6,32}$

$$
\sigma_{\mathrm{H}}^{0}=-\tau_{z} \frac{e^{2}}{2 h}\left[1-\frac{\Delta}{\mu}-\frac{\Delta\left(\mu^{2}-\Delta^{2}\right)}{\mu^{3}}\right] .
$$

Notice that the appearance of the valley index $\tau_{z}$ indicates that the Hall conductivity also takes different signs between the two valleys. In the absence of a magnetic field, the net effect is a pure valley Hall current with a vanishing charge Hall current.

When a magnetic field is turned on, from our discussion in the preceding section, there will be a field-induced valley polarization. Therefore, the Hall current from the two valleys cannot completely cancel each other and a net charge current would appear. In the low-field regime, we can calculate the charge Hall conductivity as

$$
\sigma_{\mathrm{H}}^{0 ; c}=-\frac{e^{2}}{h} \frac{\Delta}{\mu^{2}}\left(1-\frac{3 \Delta^{2}}{2 \mu^{2}}\right) \delta \mu,
$$

where $\delta \mu(\ll \mu)$ is the energy shift due to the magnetic field (the difference between the shifted band bottoms). From our discussion in Sec. II A,

$$
\delta \mu=2 m B \approx \frac{e \hbar v^{2}}{\Delta} B .
$$

Let us focus on the Hall coefficient, which is an experimentally relevant physical quantity. It is defined as 
$\gamma=-\rho_{\mathrm{H}} / B$. The Hall resistivity $\rho_{\mathrm{H}}$ is usually much less than the longitudinal resistivity $\rho$. Hence we can write $\rho_{\mathrm{H}}=\rho^{2} \sigma_{\mathrm{H}}$. Apart from the ordinary Hall coefficient from the Lorentz force $\gamma_{\mathrm{OH}}$, now we have an extra contribution $\delta \gamma^{0}$ from the valley polarization. From Eqs. (25) and (26), we have

$$
\delta \gamma^{0}(\mu ; \Delta)=\frac{1}{e \mu D(\mu)}\left(1-\frac{3 \Delta^{2}}{2 \mu^{2}}\right)\left(\frac{e^{2}}{h} \rho\right)^{2},
$$

where $D(\mu)=|\mu| /\left(2 \pi \hbar^{2} v^{2}\right)$ is the density of states for a single valley at energy $\mu$. Note that this extra contribution has a sign change at $\mu=\sqrt{3 / 2} \Delta$, which can be traced back to Eq. (24) due to the different $\mu$ dependence of the intrinsic contribution and the side-jump contribution.

Now we consider the case of TMDs. Because it consists of two copies of the massive Dirac model Eq. (1), for the $n$-doped case $(\mu>\Delta)$, the correction $\delta \gamma$ is simply a sum of the contributions from both copies,

$$
\delta \gamma=\delta \gamma^{0}(\mu-\lambda ; \Delta-\lambda)+\delta \gamma^{0}(\mu+\lambda ; \Delta+\lambda) .
$$

The situation is simpler for the weakly $p$-doped case $(-\Delta+2 \lambda>\mu>-\Delta-2 \lambda)$, where only one copy with flavor $\tau_{z} s_{z}=1$ contributes. The result is given by

$$
\delta \gamma=-\delta \gamma^{0}(-\mu+\lambda ; \Delta-\lambda) .
$$

In this case, the hole density can be estimated as

$n_{h} \simeq 2 \int_{\mu}^{-\Delta+2 \lambda} D(\varepsilon) d \varepsilon=\frac{1}{2 \pi \hbar^{2} v^{2}}(\mu-\Delta)(\mu+\Delta-2 \lambda)$,

where the factor of 2 appears because there are two valleys. The ordinary Hall coefficient is given by $\gamma_{\mathrm{OH}}=\frac{1}{n_{h} e}$. For comparison, we plot the dimensionless quantity

$$
\begin{aligned}
Q & =\frac{\delta \gamma}{\gamma_{\mathrm{OH}}} /\left(\frac{e^{2}}{h} \rho\right)^{2} \\
& =-\frac{(\mu-\Delta)(\mu+\Delta-2 \lambda)}{(\mu-\lambda)^{2}}\left[1-\frac{3(\Delta-\lambda)^{2}}{2(\mu-\lambda)^{2}}\right]
\end{aligned}
$$

in Fig. 6 as a function of the chemical potential. The sign change we noted before can be observed. Because $Q$ has a magnitude on the order of $\sim(\lambda / \Delta)$ for low doping levels $(\mu \sim$ $-\Delta)$, the correction from the valley polarization would be more important with a decreasing gap $\Delta$ and an increasing

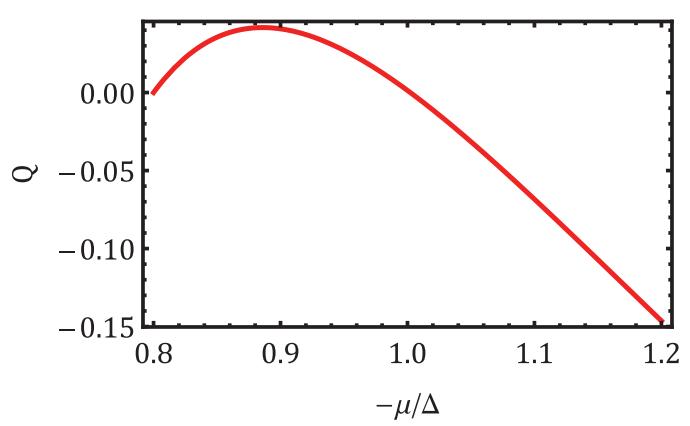

FIG. 6. (Color online) The variation of the dimensionless quantity $Q$ [see Eq. (31)] with the chemical potential $\mu$. Here, we take the spin-orbit coupling strength to be $\lambda=0.1 \Delta$ and $v=0.5 \times 10^{6} \mathrm{~m} / \mathrm{s}$. spin splitting. And it should be more pronounced for dirty samples with a large resistivity.

\section{DISCUSSION AND SUMMARY}

From the above discussions, we see that due to the valleycontrasting orbital magnetic moments and Berry curvatures, the carriers at the two valleys respond differently to the external fields. In particular, the extra contribution to the Hall transport is conceptually similar to what is happening in the anomalous Hall effect in a ferromagnet. ${ }^{46,47}$ In that case, the carriers have a net spin polarization and the transverse motion is induced by the spin-orbit coupling. In comparison, the valley here plays a similar role to the spin. A population imbalance between the two valleys is induced by the external magnetic field. We can then ask: is it possible to construct an effective coupling between the valley and the orbit motion that mimics the spinorbit coupling for the Hall transport?

On a phenomenological level, there is indeed a valley-orbit coupling, because the electrons in two valleys do have opposite transverse velocities due to the opposite Berry curvatures. The analogy with spin-orbit coupling can in fact be made more precise by a systematic and rigorous procedure for deriving an effective single-band Hamiltonian. ${ }^{48}$ There are three basic ingredients for this procedure: the band energy, the magnetic moment, and the Berry curvature. The valley explicitly comes into the effective Hamiltonian at two places. The magnetic moment carries the valley index and shifts the band energy in a magnetic field by a Zeeman-like coupling term. There is also a dipolelike term proportional to the electric field: $e \boldsymbol{E} \cdot \boldsymbol{R}$, where $\boldsymbol{R}$ is the Berry connection which represents a shift of the wave packet center. ${ }^{48}$ For the conduction band of the simple model Eq. (1), it is

$$
\boldsymbol{R}=-\frac{\tau_{z}}{2 k^{2}}\left(1-\frac{\Delta}{\sqrt{\Delta^{2}+\hbar^{2} v^{2} k^{2}}}\right) \boldsymbol{k} \times \hat{z} .
$$

Therefore, the low-energy effective Hamiltonian near the conduction-band edge can be written as

$$
\begin{aligned}
H_{\mathrm{eff}}= & \varepsilon(\boldsymbol{k})-\tau_{z} \mu_{B}^{*}(\boldsymbol{k}) \boldsymbol{B} \cdot \hat{z}-e \phi(\boldsymbol{r}) \\
& -\tau_{z} \frac{e \hbar^{2}}{4 m_{e}^{* 2} v^{2}} \hat{z} \cdot(\boldsymbol{k} \times \boldsymbol{E}),
\end{aligned}
$$

where we denote the magnitude of $\boldsymbol{m}(\boldsymbol{k})$ as an effective Bohr magneton $\mu_{B}^{*}(\boldsymbol{k})$. We can see that the preceding term has a similar form to the spin-orbit coupling, which is just the valleyorbit coupling. The effective Hamiltonian closely resembles the Pauli Hamiltonian for an electron in the nonrelativistic limit derived from the Dirac equation. Taking this effective Hamiltonian as the starting point, all the physical effects we discussed above could be addressed.

In summary, we have investigated the possibility of controlling the valley degree of freedom using an applied magnetic field. The valley-dependent orbital magnetic moment provides an essential ingredient which couples the valley index with the magnetic field. It gives a nice explanation for the asymmetric Landau level structure ${ }^{32}$ and the enhanced magnetic susceptibility that are common in systems with multiple massive Dirac fermions. 
We point out that by tuning the external magnetic field and adjusting the doping level, we can efficiently control the valley polarization. We also predict an extra contribution to the ordinary Hall effect due to the field-induced valley polarization.

For the pristine transition metal dichalcogenide material $\mathrm{MoS}_{2}$, the typical values of model parameters are $v \sim 0.53 \times$ $10^{6} \mathrm{~m} / \mathrm{s}, \Delta \sim 0.83 \mathrm{eV}$, and $\lambda \sim 0.04 \mathrm{eV}$. ${ }^{19}$ The sudden change of magnetic susceptibility at the band edge on the order of $10^{-9} \mathrm{~A} / \mathrm{T}$ should be detected. The two-step structure shown in Fig. 4 is quite a unique feature of this system. The spacing between the two steps in the valence band corresponds to the spin-orbit splitting $4 \lambda$. Hence this feature might be more clearly observed in $\mathrm{WS}_{2}$ and $\mathrm{WSe}_{2}$, which have a larger spin-orbit coupling strength, $\lambda \sim 0.1 \mathrm{eV}$. The magnetic-field-induced valley polarization could be detected through circular dichroism in optical transitions. ${ }^{21-23}$ For the weakly hole-doped case, it can also be detected by measuring the spin polarization of the carriers because the spin and valley are coupled in this case. As for both the field-induced valley polarization and the resulting additional contribution to the Hall transport, they increase as $\Delta$ decreases because both the Berry curvature and the orbital magnetic moment scale as $1 / \Delta$ near the band edge. Therefore, these effects could be enhanced if the gap can be made smaller, possibly through chemical doping, straining, or electrical gating of the two $\mathrm{S}$ or $\mathrm{Se}$ layers.

Our results presented here are particularly relevant for the valleytronics applications and for the study of $2 \mathrm{D}$ transition metal dichalcogenides materials.

\section{ACKNOWLEDGMENTS}

The authors would like to thank D. Goldhaber-Gordon and D. L. Deng for valuable discussions and inspirations.
S.A.Y. and X.L. are supported by SUTD-SRG-EPD-2013062, the MOST Project of China (2012CB921300), and NSFC (91121004). T.C. is supported by NSFC under Grant No. 11104193. F.Z. is supported by DARPA (SPAWAR N6600111-1-4110). W.Y. is supported by the HKSAR Research Grant Council under Grant No. HKU706412P and the Croucher 382 Foundation under the Croucher Innovation Award. Q.N. is supported by the Welch Foundation (F-1255) and the DOE (DE-FG03-02ER45958, Division of Materials Science and Engineering).

\section{APPENDIX: SEMICLASSICAL QUANTIZATION OF LANDAU LEVELS}

The energy of Landau levels can also be obtained by semiclassically quantizing the cyclotron orbits. The Onsager quantization condition states that the areas enclosed by the cyclotron orbits should be quantized according to ${ }^{33}$

$$
\pi k_{n}^{2}=\frac{2 \pi e B}{\hbar}\left[n+\frac{1}{2}-\frac{\Gamma\left(k_{n}\right)}{2 \pi}\right],
$$

where $n=0,1,2, \ldots$ labels the orbits and $\Gamma\left(k_{n}\right)$ is the correction from the Berry phase of the electron which is accumulated along the cyclotron orbit. Practically, it can be calculated by integrating the Berry curvature over the area enclosed by the orbit:

$$
\Gamma\left(k_{n}\right)=\int_{S_{n}} d^{2} k \boldsymbol{\Omega}(\boldsymbol{k}) \cdot \hat{z} .
$$

After we obtain the quantized values of the wave vector $k_{n}$ from Eq. (A1), the Landau level energies can be directly written down using the shifted band energy as $\varepsilon_{n}=\varepsilon\left(k_{n}\right)-m\left(k_{n}\right) B$. We have checked that the Landau levels obtained using this semiclassical approach agree very well with the exact quantum result.

\footnotetext{
*shengyuan_yang@sutd.edu.sg

${ }^{1}$ A. Rycerz, J. Tworzydlo, and C. W. J. Beenakker, Nat. Phys. 3, 172 (2007).

${ }^{2}$ D. Xiao, W. Yao, and Q. Niu, Phys. Rev. Lett. 99, 236809 (2007).

${ }^{3}$ A. R. Akhmerov and C. W. J. Beenakker, Phys. Rev. Lett. 98, 157003 (2007).

${ }^{4}$ W. Yao, D. Xiao, and Q. Niu, Phys. Rev. B 77, 235406 (2008).

${ }^{5}$ W. Yao, S. A. Yang, and Q. Niu, Phys. Rev. Lett. 102, 096801 (2009).

${ }^{6}$ F. Zhang, J. Jung, G. A. Fiete, Q. Niu, and A. H. MacDonald, Phys. Rev. Lett. 106, 156801 (2011).

${ }^{7}$ Z. Zhu, A. Collaudin, B. Fauque, W. Kang, and K. Behnia, Nat. Phys. 8, 89 (2012).

${ }^{8}$ T. Yokoyama, Phys. Rev. B 87, 241409 (2013).

${ }^{9}$ K. S. Novoselov, A. K. Geim, S. V. Morozov, D. Jiang, Y. Zhang, S. V. Dubonos, I. V. Grigorieva, and A. A. Firsov, Science 306, 666 (2004).

${ }^{10}$ K. S. Novoselov, A. K. Geim, S. V. Morozov, D. Jiang, M. I. Katsnelson, I. V. Grigorieva, S. V. Dubonos, and A. A. Firsov, Nature (London) 438, 197 (2005).
}

${ }^{11}$ Y. Zhang, Y.-W. Tan, H. L. Stormer, and P. Kim, Nature (London) 438, 201 (2005).

${ }^{12}$ B. Aufray, A. Kara, S. Vizzini, H. Oughaddou, C. Leandri, B. Ealet, and G. L. Lay, Appl. Phys. Lett. 96, 183102 (2010).

${ }^{13}$ P. E. Padova, C. Quaresima, C. Ottaviani, P. M. Sheverdyaeva, P. Moras, C. Carbone, D. Topwal, B. Olivieri, A. Kara, H. Oughaddou, B. Aufray, and G. L. Lay, Appl. Phys. Lett. 96, 261905 (2010).

${ }^{14}$ B. Lalmi, H. Oughaddou, H. Enriquez, A. Kara, S. Vizzini, B. Ealet, and B. Aufray, Appl. Phys. Lett. 97, 223109 (2010).

${ }^{15}$ K. S. Novoselov, D. Jiang, F. Schedin, T. J. Booth, V. V. Khotkevich, S. V. Morozov, and A. K. Geim, Proc. Natl. Acad. Sci. USA 102, 10451 (2005).

${ }^{16}$ A. Splendiani, L. Sun, Y. Zhang, T. Li, J. Kim, C.-Y. Chim, G. Galli, and F. Wang, Nano Lett. 10, 1271 (2010).

${ }^{17}$ K. F. Mak, C. Lee, J. Hone, J. Shan, and T. F. Heinz, Phys. Rev. Lett. 105, 136805 (2010).

${ }^{18}$ T. Korn, S. Heydrich, M. Hirmer, J. Schmutzler, and C. Schller, Appl. Phys. Lett. 99, 102109 (2011).

${ }^{19}$ D. Xiao, G.-B. Liu, W. Feng, X. Xu, and W. Yao, Phys. Rev. Lett. 108, 196802 (2012). 
${ }^{20}$ Q. H. Wang, K. Kalantar-Zadeh, A. Kis, J. N. Coleman, and M. S. Strano, Nat. Nanotech. 7, 699 (2012).

${ }^{21}$ H. Zeng, J. Dai, W. Yao, D. Xiao, and X. Cui, Nat. Nanotech. 7, 490 (2012).

${ }^{22}$ K. F. Mak, K. He, J. Shan, and T. F. Heinz, Nat. Nanotech. 7, 494 (2012).

${ }^{23}$ T. Cao, G. Wang, W. Han, H. Ye, C. Zhu, J. Shi, Q. Niu, P. Tan, E. Wang, B. Liu, and J. Feng, Nat. Commun. 3, 887 (2012).

${ }^{24}$ S. Wu, J. S. Ross, G. Liu, G. Aivazian, A. Jones, Z. Fei, W. Zhu, D. Xiao, W. Yao, D. Cobden, and X. Xu, Nat. Phys. 9, 149 (2013).

${ }^{25}$ A. M. Jones, H. Yu, N. J. Ghimire, S. Wu, G. Aivazian, J. S. Ross, B. Zhao, J. Yan, D. G. Mandrus, D. Xiao, W. Yao, and X. Xu, Nat. Nanotech. 8, 634 (2013).

${ }^{26}$ K. F. Mak, K. He, C. Lee, G. H. Lee, J. Hone, T. F. Heinz, and J. Shan, Nat. Mater. 12, 207 (2013).

${ }^{27}$ J. S. Ross, S. Wu, H. Yu, N. Ghimire, A. Jones, G. Aivazian, J. Yan, D. Mandrus, D. Xiao, W. Yao, and X. Xu, Nat. Commun. 4, 1474 (2013).

${ }^{28}$ Z. R. Gong, G. B. Liu, H. Y. Yu, D. Xiao, X. D. Cui, X. Xu, and W. Yao, Nat. Commun. 4, 2053 (2013).

${ }^{29}$ B. Radisavljevic, A. Radenovic, J. Brivio, V. Giacometti, and A. Kis, Nat. Nanotech. 6, 147 (2011).

${ }^{30}$ A. T. Neal, H. Liu, J. Gu, and P. D. Ye [Nano Lett. (to be published)], arXiv:1308.0633.

${ }^{31} \mathrm{~K}$. Bolotin (private communication).

${ }^{32}$ X. Li, F. Zhang, and Q. Niu, Phys. Rev. Lett. 110, 066803 (2013).
${ }^{33}$ M.-C. Chang and Q. Niu, Phys. Rev. B 53, 7010 (1996).

${ }^{34}$ G. Sundaram, Ph.D. thesis, The University of Texas at Austin, 2000.

${ }^{35}$ D. Xiao, M.-C. Chang, and Q. Niu, Rev. Mod. Phys. 82, 1959 (2010).

${ }^{36}$ S. G. Sharapov, V. P. Gusynin, and H. Beck, Phys. Rev. B 69, 075104 (2004).

${ }^{37}$ J. W. McClure, Phys. Rev. 104, 666 (1956).

${ }^{38}$ T. Ando, Physica E 40, 213 (2007).

${ }^{39}$ Note that in real systems, there are also contributions from the core electrons and lower-lying valence bands to the orbital susceptibility, which is not captured in the effective model. However, the sudden change at the band edge should still be visible.

${ }^{40}$ P. Marder, Condensed Matter Physics (Wiley, Hoboken, 2010).

${ }^{41}$ D. Xiao, J. Shi, and Q. Niu, Phys. Rev. Lett. 95, 137204 (2005).

${ }^{42}$ T. Jungwirth, Q. Niu, and A. H. MacDonald, Phys. Rev. Lett. 88, 207208 (2002).

${ }^{43}$ M. Onoda and N. Nagaosa, J. Phys. Soc. Jpn. 71, 19 (2002).

${ }^{44}$ L. Berger, Phys. Rev. B 2, 4559 (1970).

${ }^{45}$ For the Gaussian-type disorder, the so-called skew scattering contribution vanishes.

${ }^{46}$ N. A. Sinitsyn, J. Phys.: Condens. Matter 20, 023201 (2008).

${ }^{47}$ N. Nagaosa, J. Sinova, S. Onoda, A. H. MacDonald, and N. P. Ong, Rev. Mod. Phys. 82, 1539 (2010).

${ }^{48}$ M.-C. Chang and Q. Niu, J. Phys.: Condens. Matter 20, 193202 (2008). 